\title{
Adiponectin protects against lung ischemia-reperfusion injury in rats with type 2 diabetes mellitus
}

\author{
DI LI, LIN-LIN SONG, JUAN WANG, CHAO MENG and XIAO-GUANG CUI
}

\author{
Department of Anesthesiology, The Heilongjiang Province Key Laboratory of Research on Anesthesiology and \\ Critical Care Medicine, The Second Affiliated Hospital of Harbin Medical University, Harbin, Heilongjiang 150001, P.R. China
}

Received August 23, 2017; Accepted December 14, 2017

DOI: $10.3892 / \mathrm{mmr} .2018 .8748$

\begin{abstract}
Adiponectin (APN) has been associated with the pathogenesis of acute brain, liver and heart injury. However, the role of APN in lung ischemia-reperfusion injury (LIRI) in diabetes mellitus remains unclear. To investigate this, the present study evaluated the effects of APN on lung dysfunction and pathological alterations in rats with type 2 diabetes mellitus via lung ischemia/reperfusion (I/R). The lung-protective effects of APN globular domain (gAPN) in rats with type 2 diabetes mellitus were also investigated by measuring the oxygenation index, inflammatory cytokines, lung edema, histopathology, oxidative stress, apoptosis and the protein expression levels of phosphorylated 5'adenosine monophosphate-activated protein kinase (p-AMPK), endothelial nitric oxide synthase (eNOS) and inducible nitric oxide synthase (iNOS). The results of the present study demonstrated that the diabetes mellitus rats $+\mathrm{I} / \mathrm{R}$ (DIR) group exhibited greater concentrations of tumor necrosis factor- $\alpha$ and interleukin-6, and increases in the wet-weight to dry-weight ratio, lung injury score, oxidative stress and cellular apoptosis. These effects were accompanied by lower pulmonary oxygenation compared with the normal rat $+\mathrm{I} / \mathrm{R}$ (NIR) group $(\mathrm{P}<0.05)$. Additionally, all of these alterations were attenuated in the NIR + gAPN and DIR + gAPN groups compared with in the NIR and DIR groups, respectively. In the DIR group, the expression levels of p-AMPK/AMPK and eNOS were significantly downregulated, and the levels of iNOS were upregulated, compared with those of the NIR group. Treatment with APN activated AMPK, increased eNOS expression and attenuated iNOS expression. The results of the present study demonstrated that APN exerted protective
\end{abstract}

Correspondence to: Dr Xiao-Guang Cui, Department of Anesthesiology, The Heilongjiang Province Key Laboratory of Research on Anesthesiology and Critical Care Medicine, The Second Affiliated Hospital of Harbin Medical University, 194 Xuefu Road, Nangang, Harbin, Heilongjiang 150001, P.R. China E-mail: cuixiaoguang1018@126.com

Key words: adiponectin, diabetes mellitus, lung ischemiareperfusion injury, 5'adenosine monophosphate-activated protein kinase effects against LIRI via its anti-inflammatory, antioxidative stress and anti-apoptotic activities. These protective effects of APN were eliminated in rats with type 2 diabetes mellitus, in which LIRI was exacerbated. The present study indicated that APN may be a potential therapeutic agent for LIRI in type 2 diabetes mellitus.

\section{Introduction}

Adiponectin (APN; additionally termed Acrp30, AdipoQ and GBP28) is an adipocytokine that is secreted by adipocytes. APN has received attention due to its insulin-sensitizing effects and possible therapeutic use for metabolic disorders $(1,2)$. APN exerts antidiabetic actions by modulating glucose, fatty acid metabolism and insulin sensitivity (3-7). Apart from its antiatherosclerotic and insulin-sensitizing effects, APN additionally exhibits anti-inflammatory and antiapoptotic roles (8). Research has indicated that APN suppresses the phagocytic activity and inflammatory cytokine production of macrophages stimulated with lipopolysaccharide (9). APN additionally reduces cerebral and myocardial ischemia/reperfusion (I/R) injury, decreases proinflammatory cytokine production and reduces cellular apoptosis, and infarction of the brain and heart, via its anti-inflammatory, antioxidative stress and antiapoptotic effects $(4,10)$. It has been suggested that decreased levels of serum APN are associated with an increased risk of cardiovascular disease (CVD) development and obesity-associated types of cancer, including colon, breast, endometrial and prostate cancer (7,11-16). These results indicate that APN may possess a protective role against I/R injury that is not specific to a particular organ.

Lung I/R injury (LIRI), a primary graft dysfunction, leads to substantial morbidity and mortality following lung transplantation (LTx). Ischemia is unavoidable during LTx, and the subsequent effect of reperfusion results in marked lung inflammation, oxidative stress and cellular damage $(1,2,17)$. A study has identified that there were 1.5 million cases of mortality caused by diabetes in 2012, an increase from 1 million in 2000 (18). Diabetes has reached epidemic proportions in the general adult population of China (19). People with diabetes mellitus and prediabetes who are awaiting LTx remain at risk of mortality following LTx; donors with a history of diabetes mellitus are associated with an increased risk of mortality in the recipient following LTx $(20,21)$. 
There is increasing evidence that the lung is a target organ for diabetic microangiopathy in patients with either type 1 or type 2 diabetes mellitus $(22,23)$. Previous studies have indicated the physiological and structural abnormalities of diabetic lungs. Diabetic hyperglycemia damages the respiratory system due to pulmonary interstitial injury caused by microangiopathy, and may contribute to autonomic neuropathy (24). Decreased lung function has been associated with diabetes in cross-sectional and longitudinal studies $(25,26)$. It has additionally been reported that restrictive, although not obstructive, ventilatory dysfunction is associated with the development of prediabetes and precedes the development of type 2 diabetes mellitus (27).

A previous study reported that APN attenuated lipopolysaccharide-induced acute lung injury. Therefore, APN may have protective effects in LIRI within normal rats; the present study aimed to investigate whether APN serves a protective role LIRI in rats with diabetes mellitus.

\section{Materials and methods}

Animals. All procedures in the present study were approved by the Institutional Animal Care and Use Committee of Harbin Medical University. The animals used in the present study were supplied by the Animal Center of the Second Affiliated Hospital of Harbin Medical University (Harbin, China; no. SYXK, 2013-002). The experiments involved 36 male Wistar rats aged 8-9 weeks (200-250 g). The rats were fed with a high-fat diet or standard laboratory chow and were provided with water ad libitum. All experimental animals were housed in the same feeding environment on a 12-h light/dark cycle at a temperature of $24 \pm 2^{\circ} \mathrm{C}$ with a humidity of $60 \pm 10 \%$ prior to the establishment of the model.

Animal preparation. A total of 18 male rats were fed a high-fat diet (15\% lard, 5\% sesame oil, $20 \%$ sucrose, $2.5 \%$ cholesterol and $57.5 \%$ normal chow) for 4 weeks, followed by administration of streptozotocin (Sigma-Aldrich; Merck KGaA, Darmstadt, Germany; $35 \mathrm{mg} / \mathrm{kg}$, dissolved to $0.1 \mathrm{M}, \mathrm{pH} 4.5$ ) by intraperitoneal injection. The rats with a fasting plasma glucose $\geq 11.1 \mathrm{mmol} / 1$ (measured in blood from the tail) were considered diabetic and, therefore, as a model of type 2 diabetes mellitus (3). The control rats were fed a standard diet and injected with an equivalent volume of citrate buffer. Following anesthesia via intraperitoneal injection of $30 \mathrm{mg} / \mathrm{kg}$ sodium pentobarbital, each rat was intubated with a tracheal cannula. The rats were ventilated with $40 \% \mathrm{O}_{2}$ at 45-55 strokes $/ \mathrm{min}$ and a tidal volume of $8-10 \mathrm{ml} / \mathrm{kg}$ body weight. A 24-gauge catheter was inserted into the right femoral vein for drug and liquid administration. The right femoral artery was cannulated with a 24-gauge catheter connected to a fluid-filled pressure transducer to continuously monitor the blood pressure. The animals were positioned in the right lateral decubitus position and their chests were opened. The left lung hilum was clamped 5 min post-administration of heparin (50 IU/animal) with a non-clash microclip at the end of expiration. Subsequently, the lung was subjected to $90 \mathrm{~min}$ of ischemia and $4 \mathrm{~h}$ of reperfusion. Pentobarbital sodium was infused to maintain stable anesthesia, and rocuronium bromide was used to maintain muscle relaxation throughout the present study. Following reperfusion, blood samples were collected from the femoral artery. Subsequently, the animals were sacrificed with an overdose of pentobarbital sodium (100 mg/kg, intravenous).

Experimental protocols. All rats were randomly assigned to the normal or diabetic groups. There were three subgroups in each group; the normal groups comprised: i) Sham group (NS), in which the left lung hilum was not clamped; ii) an I/R group (NIR group), in which the lung was subjected to $90 \mathrm{~min}$ of ischemia and $4 \mathrm{~h}$ of reperfusion; and iii) an I/R + APN globular domain (gAPN) (NIRA group), in which $10 \mu \mathrm{g}$ gAPN was injected $10 \mathrm{~min}$ prior to reperfusion (5). The diabetic groups comprised: i) Sham group (DS), in which the left lung hilum was not clamped; ii) an I/R group (DIR), in which the lung was subjected to $90 \mathrm{~min}$ of ischemia and $4 \mathrm{~h}$ of reperfusion and iii) an $\mathrm{I} / \mathrm{R}+\mathrm{gAPN}$ group (DIRA), in which $10 \mu \mathrm{g}$ gAPN was injected $10 \mathrm{~min}$ prior to reperfusion.

Blood gas analysis. Arterial blood gas was measured prior to ischemia as a baseline, at $90 \mathrm{~min}$ following ischemia, and at 60 and $120 \mathrm{~min}$ following reperfusion. These time points were recorded as $\mathrm{T}_{0}-\mathrm{T}_{3}$. At the end of the experiment, blood from the femoral artery was collected and measured using a conventional analyzer (Rapid Lab 348; Bayer AG, Leverkusen, Germany).

Measurement of inflammatory cytokine levels in the bronchoalveolar lavage fluid (BALF), wet-weight to dry-weight ratio $(W / D)$ ratio and myeloperoxidase $(M P O)$ activity in the lung. The left lung was lavaged three times with $3 \mathrm{ml}$ cold sterile saline. BALF samples were centrifuged (206 x g for $10 \mathrm{~min}, 4^{\circ} \mathrm{C}$ ) and were stored at $-80^{\circ} \mathrm{C}(28)$. Interleukin (IL)-6 and tumor necrosis factor- $\alpha$ (TNF- $\alpha$ ) expression levels in the BALF were measured with ELISA kits (cat. nos. PR6000B and PRTA00, respectively; R\&D Systems, Inc., Minneapolis, MN, USA). The upper lobe of the lung graft was desiccated at $80^{\circ} \mathrm{C}$ for $72 \mathrm{~h}$ to measure the W/D ratio. A portion of the lung graft tissue was frozen, homogenized and processed for MPO detection with a colorimetric assay kit (Nanjing Jiancheng Bioengineering Institute, Nanjing, China) the change in spectrophotometric absorbance at $460 \mathrm{~nm}$ and was expressed as the optical density unit per gram of tissue.

Malondialdehyde (MDA) levels, superoxide dismutase (SOD) activity and nitric oxide (NO) production in the lung. In the lung homogenate supernatants from the left lung, MDA levels and SOD activity were determined using MDA and SOD assay kits (cat. no. A003-1 and cat. no. A001-1-1, respectively; Nanjing Jiancheng Bioengineering Institute, Nanjing, China). NO levels were measured with a commercially available kit (cat. no. A012-1 Nanjing Jiancheng Bioengineering Institute). These experiments were performed according to the manufacturers' protocols.

Histological evaluation and scoring. The middle of the left lung was immediately fixed in $10 \%$ formalin at $4^{\circ} \mathrm{C}$ for $24 \mathrm{~h}$. After $24 \mathrm{~h}$, tissues were dehydrated, embedded in paraffin, sectioned at $6 \mu \mathrm{m}$ and stained with hematoxylin and eosin for $30 \mathrm{~min}$ at room temperature. All images were acquired with a Nikon Eclipse 80i microscope (Nikon Corporation, Tokyo, Japan). Evaluation was based on the following criteria: 
i) Neutrophil infiltration; ii) airway epithelial cell damage; iii) interstitial edema; iv) hyaline membrane formation; and v) hemorrhage. Each section had five scores corresponding with the following five criteria as determined by degree of deterioration: Normal $=0$; minimal alteration $=1$; mild alteration $=2$; moderate alteration $=3$; and severe alteration $=4$. The lung injury score (LIS) for each criterion was recorded (29).

Terminal deoxynucleotidyl transferasedUTPnickend-labeling (TUNEL) staining for apoptosis. Cellular apoptosis was examined using TUNEL assays (Nanjing Jiancheng Bioengineering Institute). The lung tissues were placed in $10 \%$ formalin at room temperature overnight for paraffin embedding and then were sectioned at $5 \mu \mathrm{m}$ and were processed for TUNEL. Then they were dehydrated, incubated with $0.9 \% \mathrm{NaCl}$ for $5 \mathrm{~min}$, then rinsed with PBS for 5 min, fixed in $4 \%$ paraformaldehyde at room temperature for $15 \mathrm{~min}$, then rinsed twice with PBS for 5 min each time. The sections were mixed with biotinylated nucleotides and terminal deoxynucleotidyl transferase, covered with coverslips and incubated at $37^{\circ} \mathrm{C}$ for $60 \mathrm{~min}$. Following another PBS wash, lung tissue was blocked with $0.3 \%$ hydrogen peroxide, and incubated horseradish peroxidase streptavidin at room temperature for $30 \mathrm{~min}$, then washed 3 times with PBS and stained with hematoxylin at room temperature for $3 \mathrm{~min}$. The number of positive cells per section was counted in five random fields from every specimen with a Nikon Eclipse $80 \mathrm{i}$ microscope (magnification, $\mathrm{x} 40$; Nikon Corporation). and evaluated using the apoptotic index (AI). The AI is a measure of the number of positive cells per 100 cells counted in five different fields from the same section.

Immunohistochemistry. The lung tissues were placed in $10 \%$ formalin at room temperature overnight for paraffin embedding and were processed for immunohistochemical staining. Paraffin embedded specimens were sectioned at $5 \mu \mathrm{m}$, deparaffinized and hydrated in PBS. Then, the sections were incubated in $3 \% \mathrm{H}_{2} \mathrm{O}_{2}$ for $10 \mathrm{~min}$ and rinsed with PBS. A primary antibody against cleaved caspase-3 (cat. no. 9661; 1:200; Cell Signaling Technology, Inc., Danvers, MA, USA) was applied, followed by washing and incubation in a biotinylated secondary antibody (Vector Labs, Burlingame, CA, USA) for $30 \mathrm{~min}$. A Nikon Eclipse 80i microscope (Nikon Corporation) was used to obtain the images.

Western blot analysis. Tissues were lysed with radioimmunoprecipitation assay lysis buffer containing a protease inhibitor cocktail (Roche Diagnostics, Basel, Switzerland). Equal amounts of proteins were measured by BCA (Beyotime Institute of Biotechnology, Shanghai, China). Cell lysates with each protein (10-20 $\mu \mathrm{g} /$ well) were separated by $10-12 \%$ SDS-PAGE and transferred to nitrocellulose membranes (Pall Life Sciences, Port Washington, NY, USA). Following blocking with 5\% non-fat milk for 1.5-2 h at room temperature, the membranes were probed with primary antibodies against $\beta$-actin (cat. no. sc-47778; 1:2,000; Santa Cruz Biotechnology, Inc., Dallas, TX, USA), 5'adenosine monophosphate-activated protein kinase (AMPK; cat. no. 5831; 1:500), p-AMPK (cat. no. 2535; 1:200) endothelial nitric oxide synthase (eNOS; cat. no. 32027; 1:1,000) and inducible nitric oxide synthase (iNOS; cat. no. 13120; 1:500; all Cell Signaling
Technology, Inc.) at $4^{\circ} \mathrm{C}$ overnight. Following washing and incubating with rabbit (cat. no. 7074; 1:10,000) or mouse (cat. no. 7076; 1:10,000; both Cell Signaling Technology, Inc.) secondary antibodies at room temperature for $1 \mathrm{~h}$ in the dark, the blots were visualized using an enhanced chemiluminescence reagent (GE Healthcare Bio-Sciences, Pittsburgh, PA, USA), Intensities of blots were determined by densitometric analysis using ImageJ version 1.61 software (National Institutes of Health, Bethesda, MD, USA) and normalized to $\beta$-actin.

Statistical analyses. Statistical analyses were performed using SAS software (version 9.4; SAS Institute Inc., Cary, NC, USA). One-way or two-way analysis of variance (ANOVA) followed by the Student-Newman-Keuls or Dunnett's tests were used for the statistical comparisons between multiple groups. The non-parametric method with the Kruskal-Wallis test was used for the analysis of LIS data. $\mathrm{P}<0.05$ was considered to indicate a statistically significant with 3-6 independent experiments. Data are presented as the mean \pm standard deviation.

\section{Results}

Pulmonary oxygenation function, histological examination and lung edema. The histological structure of the alveoli was normal in the lungs of the NS group, while the lung tissues from the I/R group were markedly damaged, with intra-alveolar edema, hemorrhage and interstitial thickening. The basal membranes and alveolar walls were thickened in the rats with diabetes mellitus. These histological alterations were more notable in the DIR group and resulted in an increased cumulative LIS and W/D ratio compared with the NIR group (Fig. 1). Additionally, the partial pressure of arterial oxygen $\left(\mathrm{PaO}_{2}\right)$ /fraction of inspired oxygen $\left(\mathrm{FiO}_{2}\right)$ was decreased at $\mathrm{T}_{1}, \mathrm{~T}_{2}$ and $\mathrm{T}_{3}$ in the DIR group compared with the NIR group (Table I). Conversely, these alterations were ameliorated in the DIRA and NIRA groups compared with the DIR and NIR groups, respectively (Fig. 1). However, the LIS and W/D ratios were increased, and the $\mathrm{PaO}_{2} / \mathrm{FiO}_{2}$ was decreased at $\mathrm{T}_{2}$ and $\mathrm{T}_{3}$ in the DIRA group compared with the NIRA group (Table I).

Inflammatory cytokines and MPO activity. The expression levels of IL-6 and TNF- $\alpha$ were higher in the DS and NIR groups compared with the NS group, and the levels of IL-6 and TNF- $\alpha$ were higher in the DIR group compared with the NIR group. MPO activity exhibited the same trend as that of IL-6 expression levels. Treatment with gAPN prior to reperfusion reduced MPO activity, and IL- 6 and TNF- $\alpha$ expression levels in the NIRA and DIRA groups compared with the NIR and DIR groups. Additionally, MPO activity, and IL-6 and TNF- $\alpha$ expression levels were higher in the DIRA group compared with the NIRA group (Fig. 2).

Oxidative stress. SOD activity was decreased, and MDA and NO production levels were increased in the DS group compared with the NS group. In the NIR and DIR groups, SOD activity was significantly decreased, and the MDA and NO production levels were significantly increased compared with the NS and DS groups. In the NIRA and DIRA groups, 
Table I. Effects of gAPN on pulmonary oxygenation.

\begin{tabular}{lcccc}
\hline Group & T0 & \multicolumn{1}{c}{ T1 } & T2 & T3 \\
\hline NS & $349.7500 \pm 34.70339$ & $276.1667 \pm 18.21378$ & $321.2083 \pm 33.45647$ & $347.6250 \pm 39.13430$ \\
NIR & $340.2500 \pm 69.56634$ & $202.0417 \pm 17.91188^{\mathrm{a}}$ & $242.2500 \pm 37.09144^{\mathrm{a}}$ & $234.1250 \pm 8.93833^{\mathrm{a}}$ \\
NIRA & $341.6667 \pm 43.32974$ & $188.7083 \pm 28.02294^{\mathrm{a}}$ & $298.6667 \pm 48.61443^{\mathrm{b}}$ & $357.2500 \pm 57.97111^{\mathrm{b}}$ \\
DS & $379.5000 \pm 38.02828$ & $241.8833 \pm 21.36227^{\mathrm{a}-\mathrm{c}}$ & $298.6667 \pm 48.61443^{\mathrm{a}, \mathrm{b}}$ & $291.9583 \pm 54.47715^{\mathrm{b}}$ \\
DIR & $331.3333 \pm 67.44992$ & $161.8917 \pm 39.01699^{\mathrm{a}, \mathrm{b}, \mathrm{d}}$ & $192.2500 \pm 22.51777^{\mathrm{a}-\mathrm{d}}$ & $168.7917 \pm 22.67235^{\mathrm{a}-\mathrm{d}}$ \\
DIRA & $396.9167 \pm 100.8507$ & $159.7083 \pm 37.90957^{\mathrm{a}, \mathrm{d}, \mathrm{e}}$ & $236.3333 \pm 28.56075^{\mathrm{a}, \mathrm{c}, \mathrm{d}, \mathrm{e}}$ & $217.2500 \pm 42.53528^{\mathrm{a}, \mathrm{c}, \mathrm{d}, \mathrm{e}}$ \\
\hline
\end{tabular}

$\mathrm{T}_{0}-\mathrm{T}_{3}$ represent the following time points: Baseline, the end of ischemia, $120 \mathrm{~min}$, and $240 \mathrm{~min}$ following reperfusion, respectively. $\mathrm{PaO}_{2} / \mathrm{FiO}_{2}$, partial pressure of arterial oxygen $\left(\mathrm{PaO}_{2}\right)$ /fraction of inspired oxygen $\left(\mathrm{FiO}_{2}\right)$. Results are expressed as the mean \pm standard deviation $(\mathrm{n}=6)$. ${ }^{\mathrm{a}} \mathrm{P}<0.05$ vs. NS group, ${ }^{\mathrm{b}} \mathrm{P}<0.05$ vs. NIR group, ${ }^{\mathrm{c}} \mathrm{P}<0.05$ vs. NIRA group, ${ }^{\mathrm{d}} \mathrm{P}<0.05$ vs. $\mathrm{DS}$ group, ${ }^{\mathrm{e}} \mathrm{P}<0.05$ vs. DIR group. DIR, diabetic I/R group, in which the lung was subjected to $90 \mathrm{~min}$ of ischemia and $4 \mathrm{~h}$ of reperfusion; DIRA, diabetic I/R $+\mathrm{gAPN}$ group, in which $10 \mu \mathrm{g}$ gAPN was injected 10 min prior reperfusion; DS, diabetic sham group, in which the left lung hilum was not clamped; NIR, I/R, in which the lung was subjected to $90 \mathrm{~min}$ of ischemia and $4 \mathrm{~h}$ of reperfusion; NIRA, I/R + gAPN, in which $10 \mu \mathrm{g}$ gAPN was injected 10 min prior to reperfusion; NS, Sham group, in which the left lung hilum was not clamped. I/R, ischemia/reperfusion; gAPN, adiponectin globular domain.
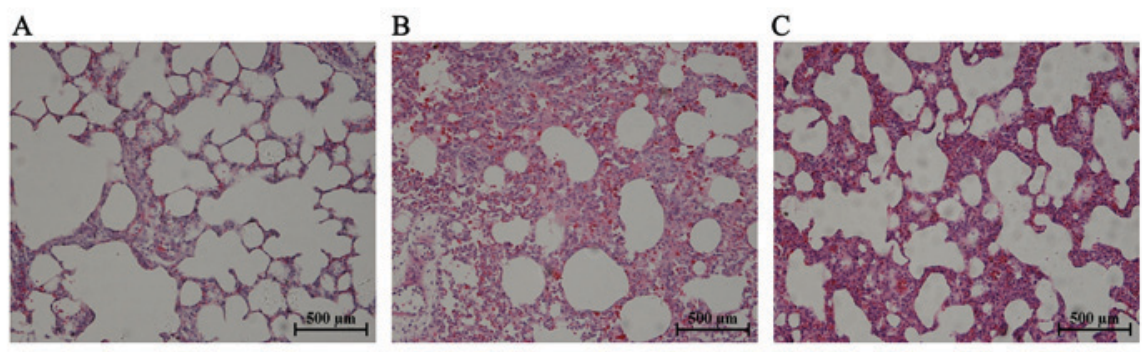

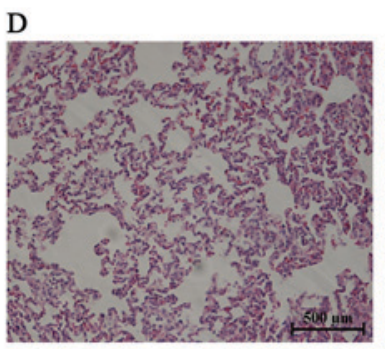

G
$\mathrm{E}$

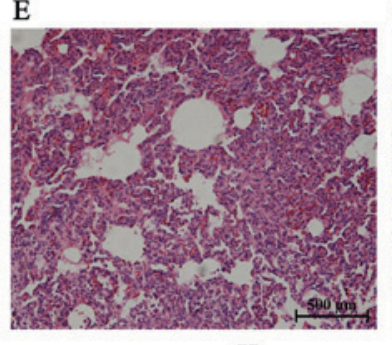

$\mathrm{H}$
$\mathrm{F}$

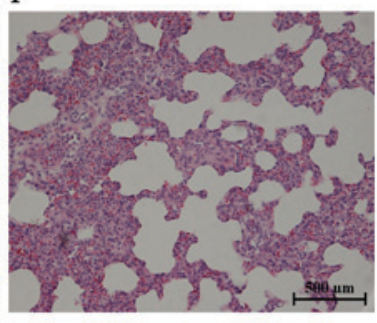

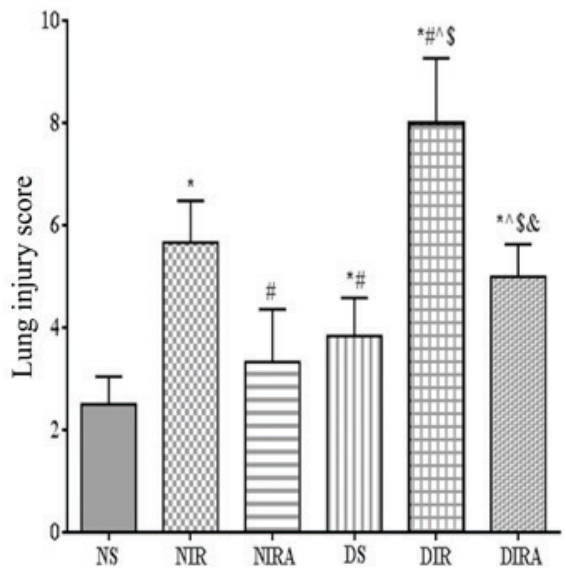

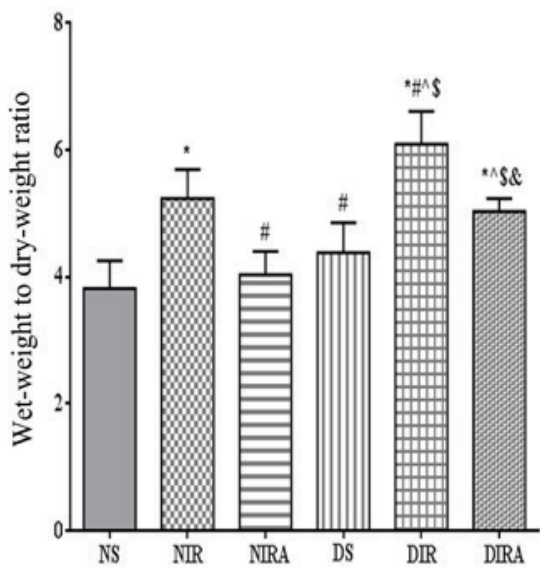

Figure 1. Diabetes mellitus increases susceptibility to lung I/R injury. Adiponectin improved the IR-induced pathological alterations in lung tissue (hematoxylin and eosin staining, lung injury score, and wet-weight to dry-weight ratio). (A) NS, (B) NIR, (C) NIRA, (D) DS, (E) DIR and (F) DIRA groups. Scale bar=500 $\mu \mathrm{M}$. (G) Lung injury score and $(\mathrm{H})$ wet-weight to dry-weight ratio were determined. The results are expressed as the mean \pm standard deviation. "P $<0.05$ vs. NS group, ${ }^{\prime} \mathrm{P}<0.05$ vs. NIR group, ${ }^{\wedge} \mathrm{P}<0.05$ vs. NIRA group, ${ }^{\$} \mathrm{P}<0.05$ vs. DS group and ${ }^{\circledR} \mathrm{P}<0.05$ vs. DIR group. DIR, diabetic I/R group, in which the lung was subjected to $90 \mathrm{~min}$ of ischemia and $4 \mathrm{~h}$ of reperfusion; DIRA, diabetic I/R $+\mathrm{gAPN}$ group, in which $10 \mu \mathrm{g}$ gAPN was injected $10 \mathrm{~min}$ prior reperfusion; DS, diabetic sham group, in which the left lung hilum was not clamped; NIR, I/R, in which the lung was subjected to 90 min of ischemia and $4 \mathrm{~h}$ of reperfusion; NIRA, I/R + gAPN, in which $10 \mu \mathrm{g}$ gAPN was injected $10 \mathrm{~min}$ prior to reperfusion; NS, Sham group, in which the left lung hilum was not clamped. I/R, ischemia/reperfusion; gAPN, adiponectin globular domain. 

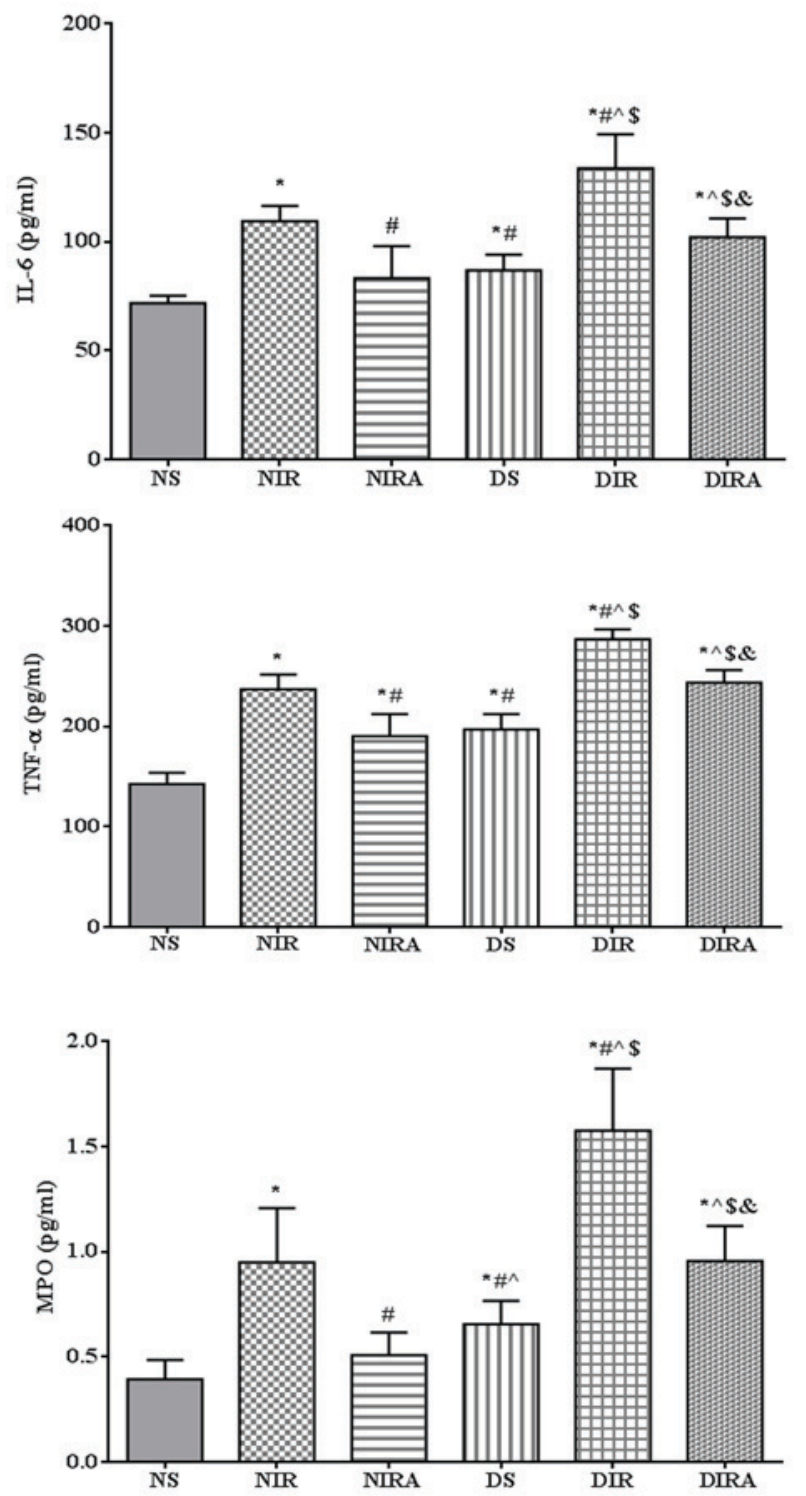

Figure 2. APN reduces the levels of IL- 6 and TNF- $\alpha$, in addition to MPO activity, following ischemia/reperfusion. The results are expressed as the mean \pm standard deviation. ${ }^{*} \mathrm{P}<0.05$ vs. NS group, ${ }^{*} \mathrm{P}<0.05$ vs. NIR group, ${ }^{\wedge} \mathrm{P}<0.05$ vs. NIRA group, ${ }^{\circ} \mathrm{P}<0.05$ vs. DS group and ${ }^{\&} \mathrm{P}<0.05$ vs. DIR group. $\mathrm{NIR}, \mathrm{I} / \mathrm{R}$, in which the lung was subjected to $90 \mathrm{~min}$ of ischemia and $4 \mathrm{~h}$ of reperfusion; NIRA, I/R + gAPN, in which $10 \mu \mathrm{g}$ gAPN was injected $10 \mathrm{~min}$ prior to reperfusion; NS, Sham group, in which the left lung hilum was not clamped; DIR, diabetic I/R group, in which the lung was subjected to $90 \mathrm{~min}$ of ischemia and $4 \mathrm{~h}$ of reperfusion; DIRA, diabetic I/R + gAPN group, in which $10 \mu \mathrm{g}$ gAPN was injected 10 min prior reperfusion; DS, diabetic sham group, in which the left lung hilum was not clamped; MPO, myeloperoxidase; TNF- $\alpha$, tumor necrosis factor- $\alpha$; gAPN, adiponectin globular domain; IL-6, interleukin-6; I/R, ischemia/reperfusion.

SOD activity was increased and MDA and NO production were decreased compared with the NIR and DIR group, respectively. SOD activity was lower, and the MDA and NO production levels were higher, in the DIRA group compared with the NIRA group (Fig. 3).

Apoptosis. The percentages of TUNEL-positive cells were increased in the DS and NIR groups compared with the NS group, and were further increased in the DIR group compared with the NIR group. Following treatment with gAPN prior
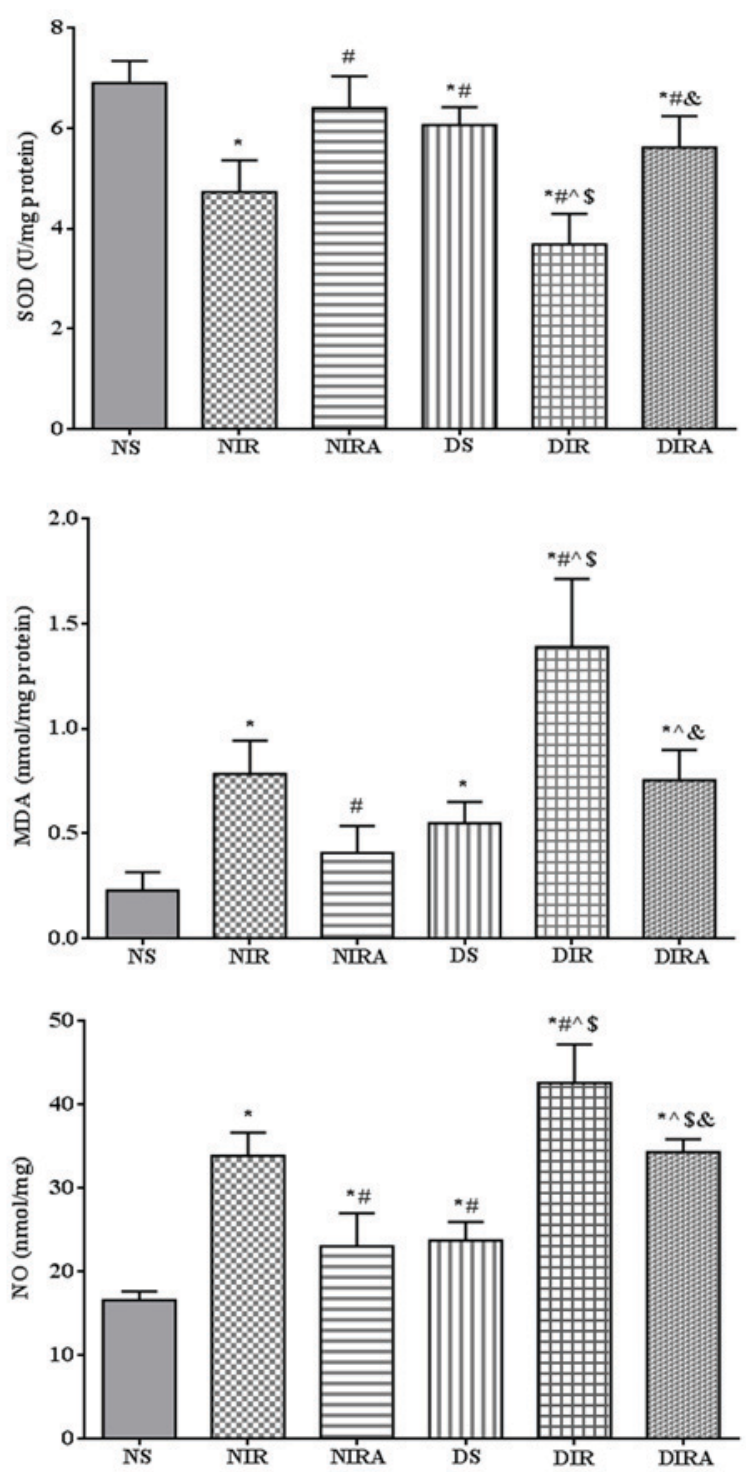

Figure 3. APN attenuates oxidative stress induced by I/R. The results are expressed as mean \pm standard deviation. ${ }^{*} \mathrm{P}<0.05$ vs. NS group, ${ }^{\#} \mathrm{P}<0.05$ vs. NIR group, ${ }^{\wedge} \mathrm{P}<0.05$ vs. NIRA group, ${ }^{\$} \mathrm{P}<0.05$ vs. $\mathrm{DS}$ group and ${ }^{\circledR} \mathrm{P}<0.05$ vs. DIR group. DIR, diabetic I/R group, in which the lung was subjected to $90 \mathrm{~min}$ of ischemia and $4 \mathrm{~h}$ of reperfusion; DIRA, diabetic I/R + gAPN group, in which $10 \mu \mathrm{g}$ gAPN was injected 10 min prior reperfusion; DS, diabetic sham group, in which the left lung hilum was not clamped; NIR, I/R, in which the lung was subjected to 90 min of ischemia and $4 \mathrm{~h}$ of reperfusion; NIRA, $\mathrm{I} / \mathrm{R}+\mathrm{gAPN}$, in which $10 \mu \mathrm{g}$ gAPN was injected $10 \mathrm{~min}$ prior to reperfusion; NS, Sham group, in which the left lung hilum was not clamped. I/R, ischemia/reperfusion; gAPN, adiponectin globular domain; SOD, superoxide dismutase; MDA, malondialdehyde; NO, nitric oxide.

to reperfusion, the percentage of TUNEL-positive cells was decreased in the DIRA and NIRA groups compared with the DIR and NIR groups (Fig. 4). The percentage of caspase-3-positive cells exhibited a pattern that was similar to that of the TUNEL-positive cells mentioned above (Fig. 5).

p-AMPK, eNOS and iNOS protein expression. The results of the present study demonstrated that p-AMPK/AMPK levels were increased in the DIR and NIR groups compared with the NS and DS groups, and were decreased in the DS group compared with the NS group (Fig. 6). Treatment with gAPN 
A

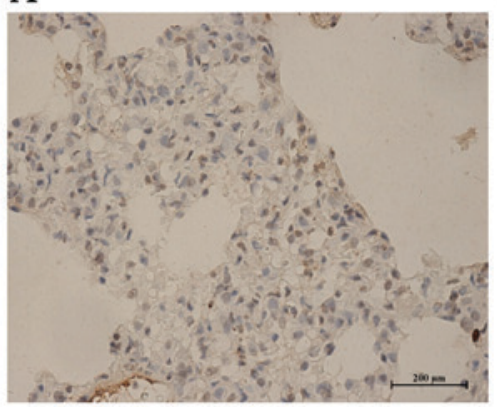

D

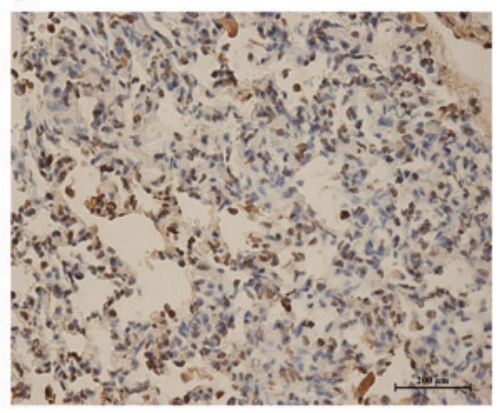

B

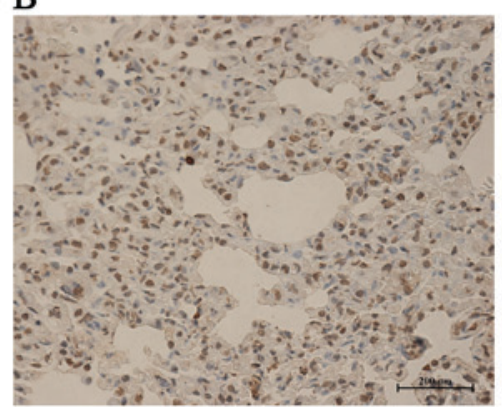

E

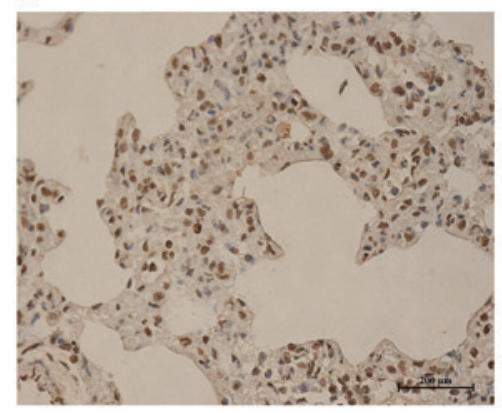

$\mathrm{C}$

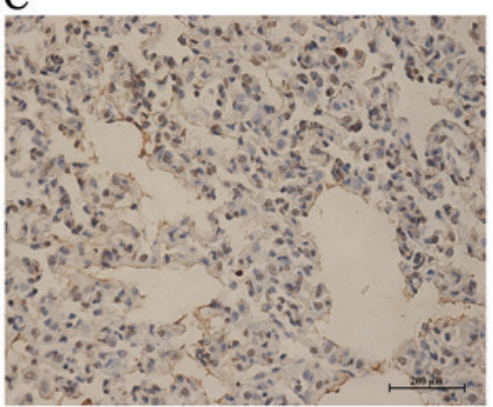

$\mathrm{F}$

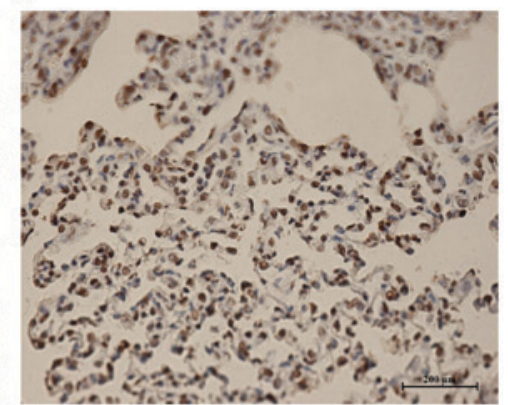

G

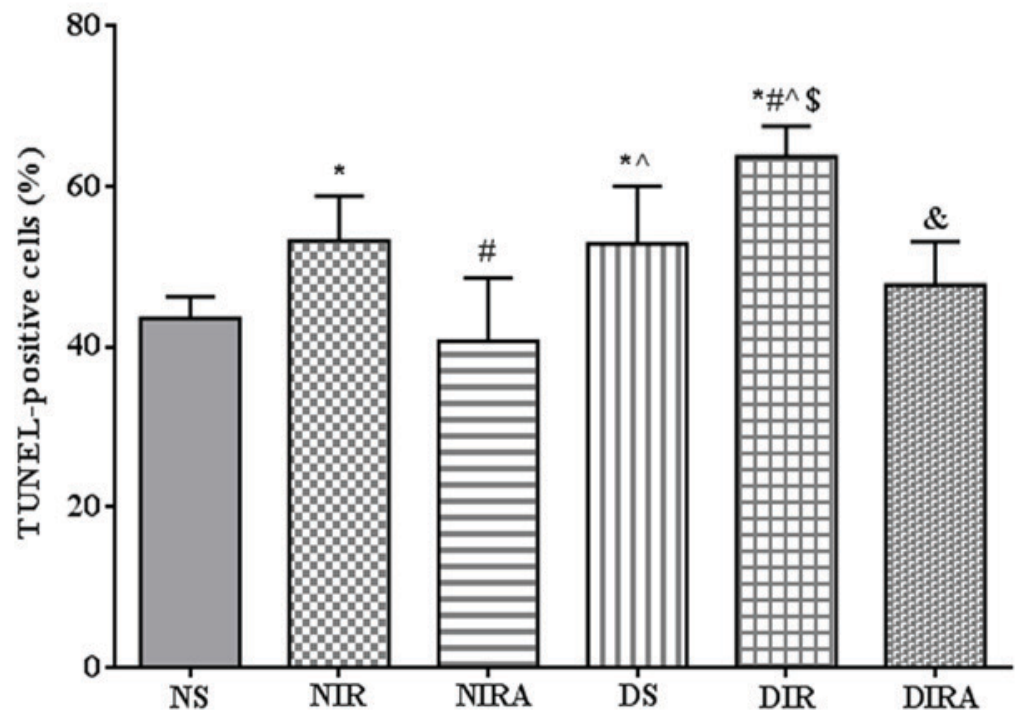

Figure 4. APN inhibits I/R-induced apoptosis, as demonstrated via TUNEL staining. TUNEL-positive cells observed under a light microscope were identified by brown-stained nuclei. (A) NS, (B) NIR, (C) NIRA, (D) DS, (E) DIR and (F) DIRA groups. Scale bar=200 $\mu$ M. (G) Percentages of TUNEL-positive cells. The results were expressed as the mean \pm standard deviation. ${ }^{*} \mathrm{P}<0.05$ vs. NS group, ${ }^{\#} \mathrm{P}<0.05$ vs. NIR group, ${ }^{\wedge} \mathrm{P}<0.05$ vs. NIRA group, ${ }^{\$} \mathrm{P}<0.05$ vs. DS group and ${ }^{\&} \mathrm{P}<0.05$ vs. DIR group. DIR, diabetic I/R group, in which the lung was subjected to 90 min of ischemia and $4 \mathrm{~h}$ of reperfusion; DIRA, diabetic I/R + gAPN group, in which $10 \mu \mathrm{g}$ gAPN was injected $10 \mathrm{~min}$ prior reperfusion; DS, diabetic sham group, in which the left lung hilum was not clamped; NIR, I/R, in which the lung was subjected to 90 min of ischemia and $4 \mathrm{~h}$ of reperfusion; NIRA, I/R + gAPN, in which $10 \mu \mathrm{g}$ gAN was injected 10 min prior to reperfusion; NS, Sham group, in which the left lung hilum was not clamped; TUNEL, terminal deoxynucleotidyl transferase dUTP nick end-labeling; I/R, ischemia/reperfusion; gAPN, adiponectin globular domain.

increased p-AMPK/AMPK levels in the NIRA and DIRA groups compared with the NIR and DIR groups. Additionally, p-AMPK/AMPK levels were decreased in the DIRA group compared with the NIRA group. The eNOS levels exhibited a pattern that was similar to that of $\mathrm{p}$-AMPK/AMPK, whereas iNOS levels exhibited a trend that was the inverse of that of p-AMPK/AMPK (Fig. 6).

\section{Discussion}

The present study reported three primary findings: i) Type 2 diabetes mellitus exacerbates LIRI in a manner that is characterized by a high level of inflammatory cytokines, and a large increase in oxidative stress and cellular apoptosis; ii) conditioning via pretreatment with gAPN alleviates LIRI in vivo 
A

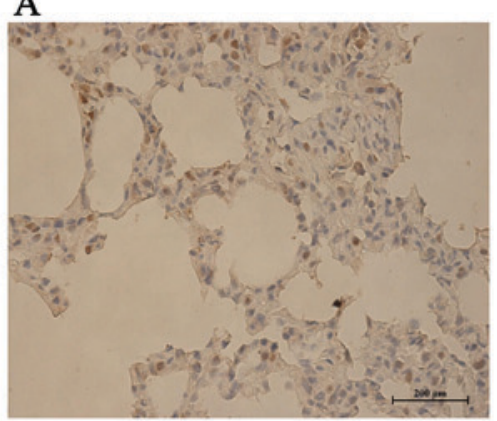

D

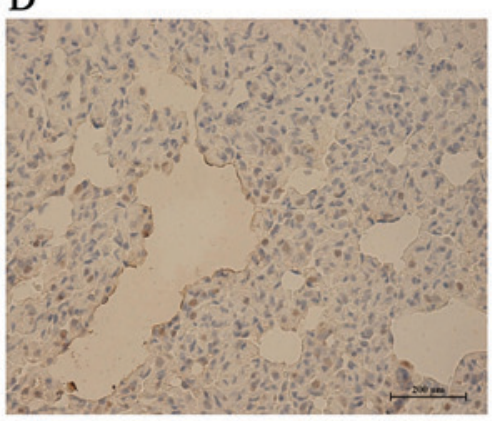

B

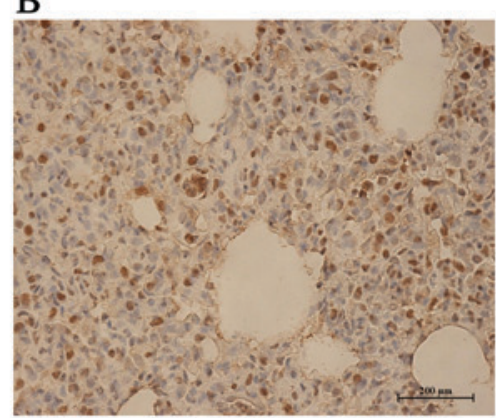

E

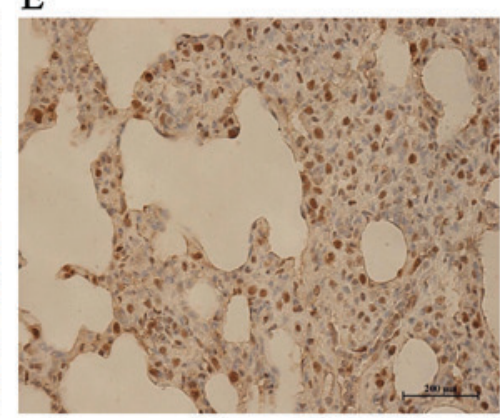

C

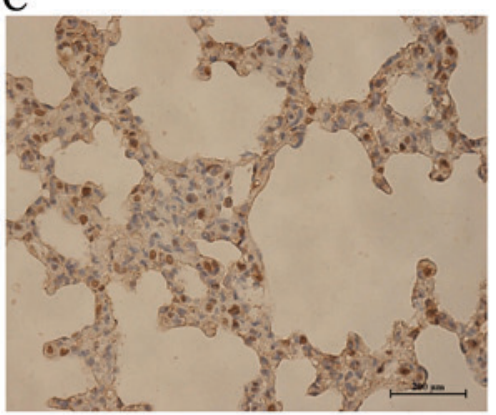

F

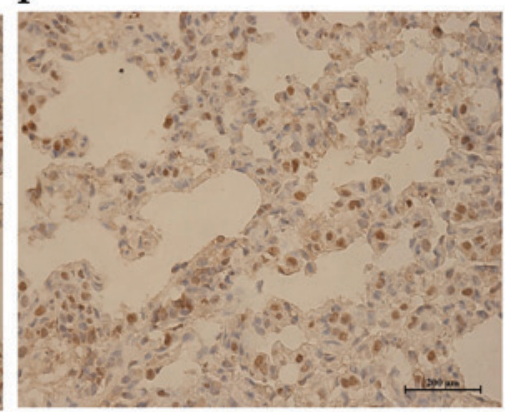

G

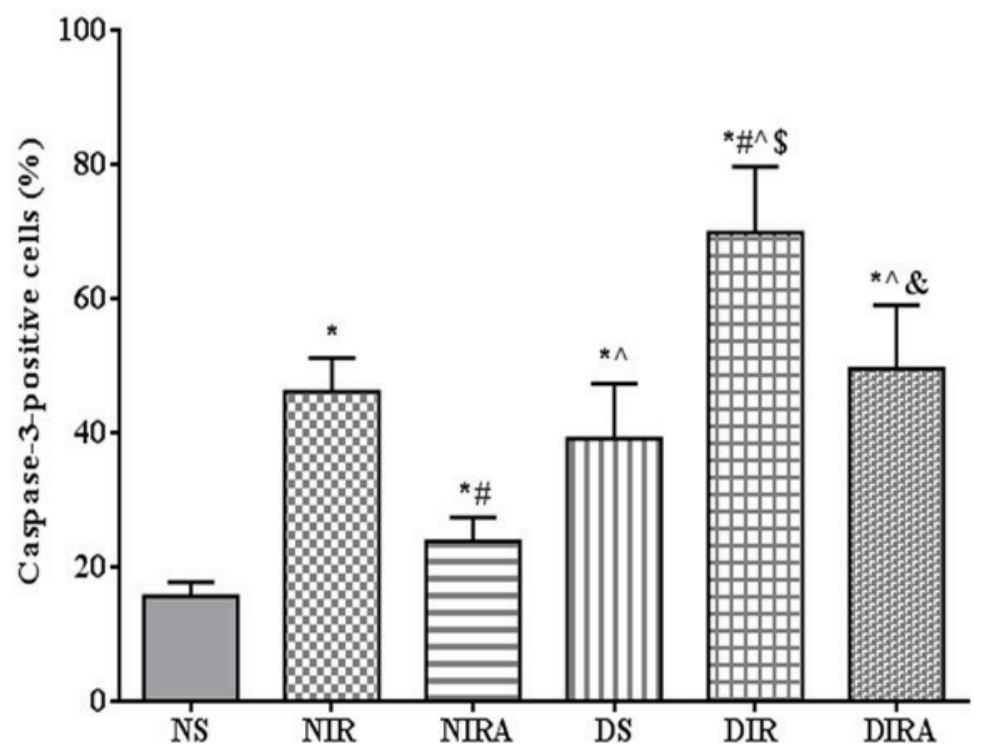

Figure 5. APN inhibits I/R-induced caspase-3 expression. Caspase-3-positive cells observed under a light microscope were identified by brown-stained nuclei. (A) NS, (B) NIR, (C) NIRA, (D) DS, (E) DIR and (F) DIRA groups. Scale bar=200 $\mu$ M. (G) Percentages of caspase-3-positive cells. The results are expressed as the mean \pm standard deviation. ${ }^{*} \mathrm{P}<0.05$ vs. NS group, ${ }^{*} \mathrm{P}<0.05$ vs. NIR group, ${ }^{\wedge} \mathrm{P}<0.05$ vs. NIRA group, ${ }^{\$} \mathrm{P}<0.05$ vs. $\mathrm{DS}$ group and ${ }^{\circledR} \mathrm{P}<0.05$ vs. DIR group. DIR, diabetic I/R group, in which the lung was subjected to $90 \mathrm{~min}$ of ischemia and $4 \mathrm{~h}$ of reperfusion; DIRA, diabetic I/R $+\mathrm{gAPN}$ group, in which $10 \mu \mathrm{g}$ gAPN was injected $10 \mathrm{~min}$ prior reperfusion; DS, diabetic sham group, in which the left lung hilum was not clamped; NIR, I/R, in which the lung was subjected to $90 \mathrm{~min}$ of ischemia and $4 \mathrm{~h}$ of reperfusion; NIRA, I/R + gAPN, in which $10 \mu \mathrm{g}$ gAPN was injected $10 \mathrm{~min}$ prior to reperfusion; NS, Sham group, in which the left lung hilum was not clamped. I/R, ischemia/reperfusion; gAPN, adiponectin globular domain.

by reducing the inflammatory response, lung edema, LIS, oxidative stress and apoptosis, and by improving pulmonary oxygenation in normal rats and DM rats; and iii) treatment with gAPN increases the activation of AMPK in rats, regardless of treatment. To the best of our knowledge, the present study was the first to provide direct evidence that the administration of gAPN as a bolus 10 min prior to reperfusion reverses the adverse effects of type 2 diabetes on LIRI.
APN monomers possess an amino-terminal collagen-like domain and a carboxy-terminal globular domain that generates trimers, hexamers and high-molecular-weight multimers (30). The three multimeric forms have been detected in the circulation, associated with numerous serum proteins previously characterized in humans (31). The APN globular head has additionally been detected in the trimeric form in human and mouse plasma, albeit at low concentrations $(32,33)$. APN is one of a 

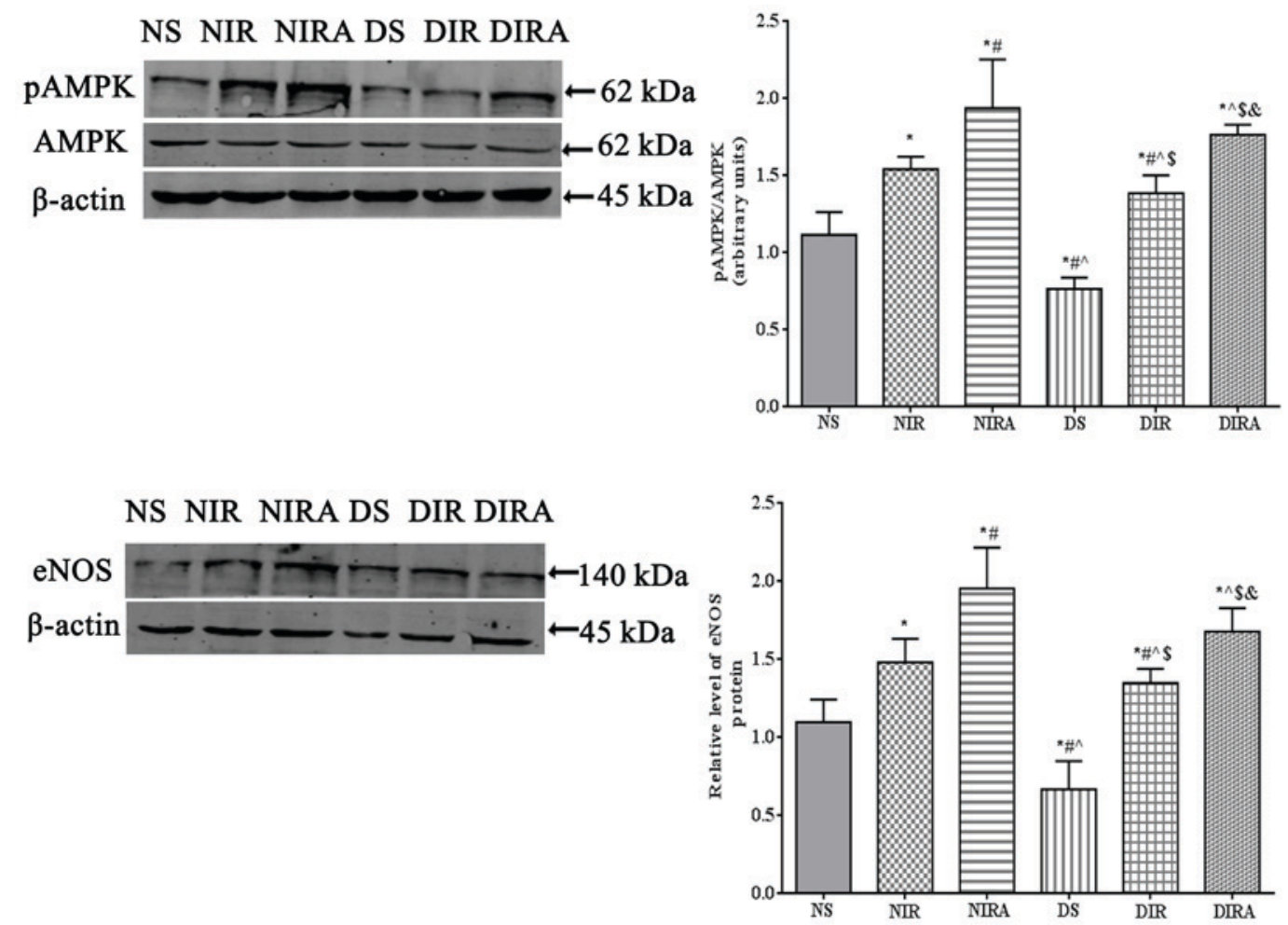

NS NIR NIRA DS DIR DIRA
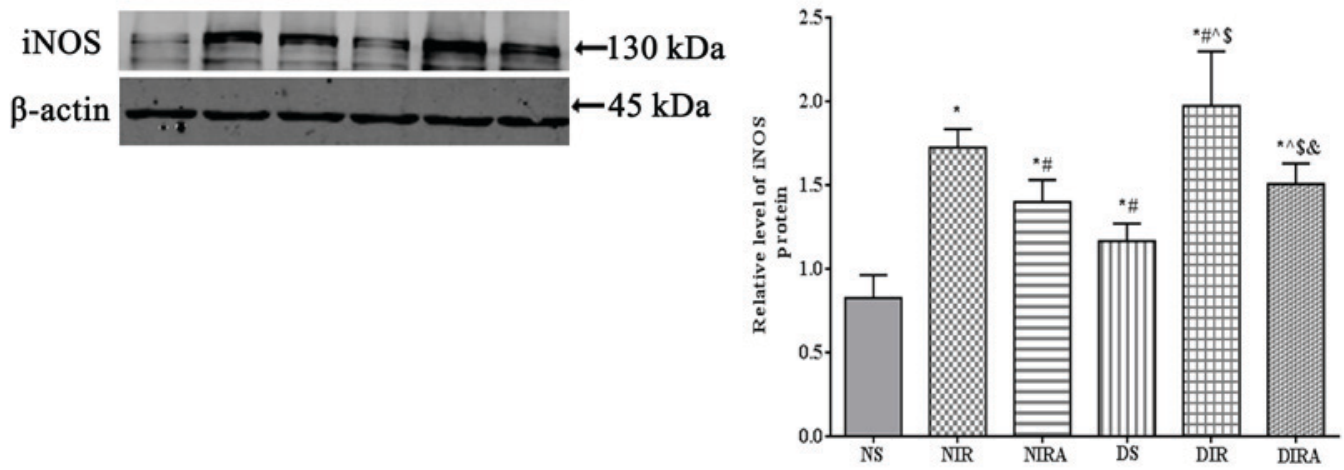

Figure 6. APN activates p-AMPK and eNOS expression and attenuates iNOS expression. The results are expressed as mean \pm standard deviation. ${ }^{*} \mathrm{P}<0.05$ vs. NS group, ${ }^{\#} \mathrm{P}<0.05$ vs. NIR group, ${ }^{\wedge} \mathrm{P}<0.05$ vs. NIRA group, ${ }^{\circ} \mathrm{P}<0.05$ vs. DS group and ${ }^{\circledR} \mathrm{P}<0.05$ vs. DIR group. DIR, diabetic I/R group, in which the lung was subjected to $90 \mathrm{~min}$ of ischemia and $4 \mathrm{~h}$ of reperfusion; DIRA, diabetic I/R + gAPN group, in which $10 \mu \mathrm{g}$ gAPN was injected 10 min prior reperfusion; DS, diabetic sham group, in which the left lung hilum was not clamped; NIR, I/R, in which the lung was subjected to 90 min of ischemia and $4 \mathrm{~h}$ of reperfusion; NIRA, I/R + gAPN, in which $10 \mu \mathrm{g}$ gAPN was injected 10 min prior to reperfusion; NS, Sham group, in which the left lung hilum was not clamped. p-AMPK, phosphorylated 5' adenosine monophosphate-activated protein kinase; I/R, ischemia/reperfusion; gAPN, adiponectin globular domain; eNOS, endothelial nitric oxide synthase; iNOS, inducible nitric oxide synthase.

number of proteins secreted by adipose cells that may couple the regulation of insulin sensitivity with energy metabolism and serve to associate obesity with insulin resistance (34). It has been reported that gAPN has increased biological activity ( $>20$-fold) compared with the full-length form $(33,35)$. gAPN is more potent in the stimulation of fatty acid oxidation in skeletal muscles compared with full-length APN and has more rapid action than full-length APN following a single in vivo dose (32). In addition, Tao et al (4) reported that, although the levels of gAPN may be markedly low in the circulation, gAPN may be the final active ligand of APN at its target cells.

It has been demonstrated that APN decreases proinflammatory cytokine levels and reduces apoptosis and oxidative/nitrative stress $(4,5,10)$. In the present study, the
LIRI of normal rats was mitigated following administration of APN. Compared with the NIRA group, the W/D ratio, LIS, IL- 6 expression levels, TNF- $\alpha$ expression levels and MPO activity were increased; oxidative stress and apoptosis were more severe in the NIR group. Therefore, APN had a protective effect on cerebral and myocardial I/R injury $(10,36)$, in addition to LIRI. Low levels of APN may increase the risk of type 2 diabetes mellitus and CVD development; however, the association between circulating APN concentrations and CVD risk is moderate compared with the strong association with type 2 diabetes mellitus risk (6). In the present study, diabetes mellitus in rats induced more severe lung injury and aggravated LIRI compared with normal rats in the same state. Compared with the NIR group, the W/D ratio, 
LIS, IL-6 expression levels, TNF- $\alpha$ expression levels and MPO activity were higher; oxidative stress and apoptosis were more severe in the DIR group. These changes were ameliorated in the DIRA group compared with the DIR group. Therefore, the administration of exogenous APN mitigated LIRI in rats with diabetes mellitus. Additionally, compared with the NIRA group, the W/D ratio, LIS, IL-6 expression levels, TNF- $\alpha$ expression levels and MPO activity were higher; oxidative stress and apoptosis were more severe within the DIRA group. Therefore, APN had a protective effect on LIRI, and this protective effect was inhibited in rats with diabetes mellitus.

AMPK is a highly conserved heterotrimeric kinase that functions as a metabolic switch, thereby coordinating the cellular enzymes involved in carbohydrate and fat metabolism to enable adenosine 5'-triphosphate conservation and synthesis (37). The metabolic effects of APN are similar to those elicited by the activation of AMPK in the liver and muscles $(7,11,12)$, leading to the hypothesis that APN may act via the stimulation of this enzyme. AMPK signaling in endothelial cells is required for the proangiogenic effects of APN $(12,38)$, antiapoptotic effects (39), stimulation of NO production (40), reduction of myocardial infarct size and myocardial apoptosis in a mouse model of heart I/R (36), and attenuation of acute lung injury $(41,42)$. The majority of the metabolic regulatory function of APN occurs via the AMPK signaling axis (35), whereas APN-mediated inhibition of the inflammatory response and elicitation of vasodilatation/vasculoprotection occurs primarily via the AMPK/eNOS axis (38-40). Previous research revealed that AMPK activation reduces TLR4-induced neutrophil activation and diminishes the severity of neutrophil-driven proinflammatory processes, including acute lung injury (43). AMPK is involved in modulating acute inflammatory reactions and AMPK activation inhibited cytokine production (44). AMPK may additionally inhibit the formation of reactive oxygen species via NADPH oxidase and stimulate NO production by eNOS $(45,46)$. Consistent with these studies, the present study demonstrated that APN activated AMPK expression, protected against LIRI by countering inflammation, inhibited oxidative stress and inhibited apoptosis in rats. In type 2 diabetes mellitus, tissue responses to insulin are significantly reduced, and numerous studies have demonstrated that APN shares numerous biological functions with insulin (47). Therefore, rats with diabetes mellitus may exhibit downregulated phosphorylation of AMPK. In the present study, p-AMPK/AMPK was decreased in diabetes mellitus rats compared with normal rats under the same conditions.

APN directly stimulates NO production via the phosphorylation of eNOS by AMPK (48). In the present study, treatment with gAPN decreased NO production; however, eNOS expression was increased after treatment with gAPN. In contrast to eNOS expression, iNOS expression was enhanced following I/R and treatment with gAPN inhibited iNOS expression. A previous study suggested that APN may increase NO production by eNOS under physiological conditions; whereas, under pathological conditions, where iNOS expression is stimulated, APN inhibits NO overproduction by inhibiting iNOS expression, thus protecting tissues $(5,48)$. These observations are consistent with those of the present study, in which treatment with gAPN decreased NO production and iNOS expression in the NIRA and DIRA groups compared with the NIR and DIR groups, respectively.

Overall, the present study suggested that APN may serve an important protective role in diabetes mellitus-exacerbated LIRI in rats. Further studies are required to clarify the underlying mechanisms through which APN may protect against diabetes mellitus-exacerbated LIRI in rats.

There were a number of limitations to the present study; as the present study was terminated $4 \mathrm{~h}$ subsequent to reperfusion, extended observations of the recipients of APN are required, and the therapeutic effects of pretreatment with APN on lung function requires further investigation. In the present study, normal rats and diabetes mellitus rats were administered the same dose of APN. The protective effects of APN were eliminated in diabetes mellitus rats; an increased dose of APN may exhibit more protective effects within diabetes mellitus rats. An APN knockout mouse model is required to investigate the underlying mechanisms of action of APN.

The results of the present study demonstrated that gAPN may exert potent protective functions against LIRI within rats with type 2 diabetes mellitus and the anti-inflammatory, antioxidative stress and antiapoptotic properties of APN may contribute considerably to its beneficial effects. AMPK is a key transcription factor in this process. These molecules may be considered as potential novel therapeutic targets following LTx.

\section{Acknowledgements}

Not applicable.

\section{Funding}

No funding was received.

\section{Availability of data and materials}

All data generated or analyzed during this study are included in this published article.

\section{Authors' contributions}

DL and XC designed the present study. DL performed the experiments, collected and analyzed data. LS, JW and CM provided experimental support. DL wrote the manuscript and JW, LS and XC proofread the manuscript. All authors read and approved the final manuscript.

\section{Ethics approval and consent to participate}

The protocol for the present study was approved by Institutional Committee on Animal Care and Use of Harbin Medical University (Harbin, China).

\section{Consent for publication}

Not applicable.

\section{Competing interests}

The authors declare that they have no competing interests. 


\section{References}

1. Fernandez LG, Sharma AK, LaPar DJ, Kron IL and Laubach VE: Adenosine A1 receptor activation attenuates lung ischemia-reperfusion injury. J Thorac Cardiovasc Surg 145: 1654-1659, 2013

2. den Hengst WA, Gielis JF, Lin JY, Van Schil PE, De Windt LJ and Moens AL: Lung ischemia-reperfusion injury: A molecular and clinical view on a complex pathophysiological process. Am J Physiol Heart Circ Physiol 299: H1283-H1299, 2010.

3. Chen GM, Hu N, Liu L, Xie SS, Wang P, Li J, Xie L, Wang GJ and Liu XD: Pharmacokinetics of verapamil in diabetic rats induced by combination of high-fat diet and streptozotocin injection. Xenobiotica 41: 494-500, 2011.

4. Tao L, Gao E, Jiao X, Yuan Y, Li S, Christopher TA, Lopez BL, Koch W, Chan L, Goldstein BJ and Ma XL: Adiponectin cardioprotection after myocardial ischemia/reperfusion involves the reduction of oxidative/nitrative stress. Circulation 115: 1408-1416, 2007.

5. Yi W, Sun Y, Gao E, Wei X, Lau WB, Zheng Q, Wang Y, Yuan Y, Wang X, Tao L, et al: Reduced cardioprotective action of adiponectin in high-fat diet-induced type II diabetic mice and its underlying mechanisms. Antioxid Redox Signal 15: 1779-1788, 2011.

6. Sattar N, Wannamethee G, Sarwar N, Tchernova J, Cherry L, Wallace AM, Danesh J and Whincup PH: Adiponectin and coronary heart disease: A prospective study and meta-analysis. Circulation 114: 623-629, 2006.

7. Kahn BB, Alquier T, Carling D and Hardie DG: AMP-activated protein kinase: Ancient energy gauge provides clues to modern understanding of metabolism. Cell Metab 1: 15-25, 2005.

8. Whitehead JP, Richards AA, Hickman IJ, Macdonald GA and Prins JB: Adiponectin-a key adipokine in the metabolic syndrome. Diabetes Obes Metab 8: 264-280, 2006.

9. Thakur V, Pritchard MT, McMullen MR and Nagy LE: Adiponectin normalizes LPS-stimulated TNF-alpha production by rat Kupffer cells after chronic ethanol feeding. Am J Physiol Gastrointest Liver Physiol 290: G998-G1007, 2006.

10. Chen B, Liao WQ, Xu N, Xu H, Wen JY, Yu CA, Liu XY, Li CL, Zhao SM and Campbell W: Adiponectin protects against cerebral ischemia-reperfusion injury through anti-inflammatory action. Brain Res 1273: 129-137, 2009.

11. Kola B, Boscaro M, Rutter GA, Grossman AB and Korbonits M Expanding role of AMPK in endocrinology. Trends Endocrinol Metab 17: 205-215, 2006.

12. Viollet B, Foretz M, Guigas B, Horman S, Dentin R, Bertrand L, Hue L and Andreelli F: Activation of AMP-activated protein kinase in the liver: A new strategy for the management of metabolic hepatic disorders. J Physiol 574: 41-53, 2006.

13. Ouchi N, Kobayashi H, Kihara S, Kumada M, Sato K, Inoue T, Funahashi $\mathrm{T}$ and Walsh K: Adiponectin stimulates angiogenesis by promoting cross-talk between AMP-activated protein kinase and Akt signaling in endothelial cells. J Biol Chem 279: 1304-1309, 2004.

14. Ouchi N, Kihara S, Arita Y, Maeda K, Kuriyama H, Okamoto Y, Hotta K, Nishida M, Takahashi M, Nakamura T, et al: Novel modulator for endothelial adhesion molecules: Adipocyte-derived plasma protein adiponectin. Circulation 100 2473-2476, 1999.

15. Kumada M, Kihara S, Sumitsuji S, Kawamoto T, Matsumoto S Ouchi N, Arita Y, Okamoto Y, Shimomura I, Hiraoka H, et al: Association of hypoadiponectinemia with coronary artery disease in men. Arterioscler Thromb Vasc Biol 23: 85-89, 2003

16. Bouhali T, Brisson D, St-Pierre J, Tremblay G,Perron P, Laprise C, Vohl MC, Vissers MN, Hutten BA, Després JP, et al: Low plasma adiponectin exacerbates the risk of premature coronary artery disease in familial hypercholesterolemia. Atherosclerosis 196 : 262-269, 2008

17. Laubach VE and IL Kron: Pulmonary inflammation after lung transplantation. Surgery 146: 1-4, 2009.

18. Zheng $\mathrm{H}$, Wu J, Jin $\mathrm{Z}$ and Yan LJ: Potential biochemical mechanisms of lung injury in diabetes. Aging Dis 8: 7-16, 2017.

19. Yang W, Lu J, Weng J, Jia W, Ji L, Xiao J, Shan Z, Liu J, Tian H, Ji Q, et al: Prevalence of diabetes among men and women in China. N Engl J Med 362: 1090-1101, 2010.

20. Hackman KL, Snell GI and Bach LA: An unexpectedly high prevalence of undiagnosed diabetes in patients awaiting lung transplantation. J Heart Lung Transplant 32: 86-91, 2013.
21. Ambur V, Taghavi S, Jayarajan S, Kadakia S, Zhao $\mathrm{H}$, Gomez-Abraham J and Toyoda Y: The impact of lungs from diabetic donors on lung transplant recipientst. Eur J Cardiothorac Surg 51: 285-290, 2017.

22. Kuitert LM: The lung in diabetes-yet another target organ? Chron Respir Dis 5: 67-68, 2008.

23. Pitocco D, Fuso L, Conte EG, Zaccardi F, Condoluci C, Scavone G, Incalzi RA and Ghirlanda G: The diabetic lung-a new target organ? Rev Diabet Stud 9: 23-35, 2012.

24. Williams JG, Morris AI, Hayter RC and Ogilvie CM: Respiratory responses of diabetics to hypoxia, hypercapnia, and exercise. Thorax 39: 529-534, 1984 .

25. Dennis RJ, Maldonado D, Rojas MX, Aschner P, Rondón M, Charry L and Casas A: Inadequate glucose control in type 2 diabetes is associated with impaired lung function and systemic inflammation: A cross-sectional study. BMC Pulm Med 10: 38 , 2010.

26. Engström G, Hedblad B, Nilsson P, Wollmer P, Berglund G and Janzon L: Lung function, insulin resistance and incidence of cardiovascular disease: A longitudinal cohort study. J Intern Med 253: 574-581, 2003.

27. Kim CH, Kim HK, Kim EH, Bae SJ, Jung YJ, Choi J and Park JY: Association of restrictive ventilatory dysfunction with the development of prediabetes and type 2 diabetes in Koreans. Acta Diabetol 52: 357-363, 2015.

28. Chiang $\mathrm{CH}$, Hsu K, Yan HC, Harn HJ and Chang DM: PGE1, dexamethasone, U-74389G, or Bt2-cAMP as an additive to promote protection by UW solution in I/R injury. J Appl Physiol (1985) 83: 583-590, 1997.

29. Pirat A, Zeyneloglu P, Aldemir D, Yücel M, Ozen O, Candan S and Arslan G: Pretreatment with simvastatin reduces lung injury related to intestinal ischemia-reperfusion in rats. Anesth Analg 102: 225-232, 2006.

30. Kadowaki T, Yamauchi T, Kubota N, Hara K, Ueki K and Tobe K: Adiponectin and adiponectin receptors in insulin resistance, diabetes, and the metabolic syndrome. J Clin Invest 116: 784-792, 2006.

31. Wang Y, Xu LY, Lam KS, Lu G, Cooper GJ and Xu A: Proteomic characterization of human serum proteins associated with the fat-derived hormone adiponectin. Proteomics 6: 3862-3870, 2006.

32. Fruebis J, Tsao TS, Javorschi S, Ebbets-Reed D, Erickson MR, Yen FT, Bihain BE and Lodish HF: Proteolytic cleavage product of $30-\mathrm{kDa}$ adipocyte complement-related protein increases fatty acid oxidation in muscle and causes weight loss in mice. Proc Natl Acad Sci USA 98: 2005-2010, 2001.

33. Yamauchi T, Kamon J, Waki H, Terauchi Y, Kubota N, Hara K, Mori Y, Ide T, Murakami K, Tsuboyama-Kasaoka N, et al: The fat-derived hormone adiponectin reverses insulin resistance associated with both lipoatrophy and obesity. Nat Med 7: 941-946, 2001.

34. Iwashima Y, Katsuya T, Ishikawa K, Ouchi N, Ohishi M, Sugimoto K, Fu Y, Motone M, Yamamoto K, Matsuo A, et al: Hypoadiponectinemia is an independent risk factor for hypertension. Hypertension 43: 1318-1323, 2004.

35. Yamauchi T, Kamon J, Minokoshi Y, Ito Y, Waki H, Uchida S, Yamashita S, Noda M, Kita S, Ueki K, et al: Adiponectin stimulates glucose utilization and fatty-acid oxidation by activating AMP-activated protein kinase. Nat Med 8: 1288-1295, 2002.

36. Shibata R, Sato K, Pimentel DR, Takemura Y, Kihara S, Ohashi K, Funahashi T, Ouchi N and Walsh K: Adiponectin protects against myocardial ischemia-reperfusion injury through AMPK- and COX-2-dependent mechanisms. Nat Med 11: 1096-1103, 2005.

37. Hardie DG, Carling D and Carlson M: The AMP-activated/SNF1 protein kinase subfamily: Metabolic sensors of the eukaryotic cell? Annu Rev Biochem 67: 821-855, 1998.

38. Shibata R, Ouchi N, Kihara S, Sato K, Funahashi T and Walsh K Adiponectin stimulates angiogenesis in response to tissue ischemia through stimulation of amp-activated protein kinase signaling. J Biol Chem 279: 28670-28674, 2004.

39. Kobayashi H, Ouchi N, Kihara S, Walsh K, Kumada M, Abe Y, Funahashi T and Matsuzawa Y: Selective suppression of endothelial cell apoptosis by the high molecular weight form of adiponectin. Circ Res 94: e27-e31, 2004.

40. Chen H, Montagnani M, Funahashi T, Shimomura I and Quon MJ: Adiponectin stimulates production of nitric oxide in vascular endothelial cells. J Biol Chem 278: 45021-45026, 2003. 
41. Konter JM,Parker JL, Baez E, Li SZ, Ranscht B, Denzel M, Little FF, Nakamura K, Ouchi N, Fine A, et al: Adiponectin attenuates lipopolysaccharide-induced acute lung injury through suppression of endothelial cell activation. J Immunol 188: 854-863, 2012.

42. He Y, Zou L, Zhou Y, Hu H, Yao R, Jiang Y, Lau WB, Yuan T, Huang W, Zeng Z and Cao Y: Adiponectin ameliorates the apoptotic effects of paraquat on alveolar type cells via improvements in mitochondrial function. Mol Med Rep 14: 746-752, 2016.

43. Zhao X, Zmijewski JW, Lorne E, Liu G, Park YJ, Tsuruta Y and Abraham E: Activation of AMPK attenuates neutrophil proinflammatory activity and decreases the severity of acute lung injury. Am J Physiol Lung Cell Mol Physiol 295: L497-L504, 2008.

44. Becker J, Delayre-Orthez C, Frossard N and Pons F: Regulation of inflammation by PPARs: A future approach to treat lung inflammatory diseases? Fundam Clin Pharmacol 20: 429-447, 2006.

45. Alba G, El Bekay R, Alvarez-Maqueda M, Chacón P, Vega A, Monteseirín J, Santa María C, Pintado E, Bedoya FJ, Bartrons R and Sobrino F: Stimulators of AMP-activated protein kinase inhibit the respiratory burst in human neutrophils. FEBS Lett 573: 219-225, 2004.
46. Song $\mathrm{P}$ and Zou MH: Regulation of NAD(P)H oxidases by AMPK in cardiovascular systems. Free Radic Biol Med 52: $1607-1619,2012$.

47. Berg AH, Combs TP, Du X, Brownlee M and Scherer PE: The adipocyte-secreted protein Acrp30 enhances hepatic insulin action. Nat Med 7: 947-953, 2001.

48. Huang PH, Sata M, Nishimatsu H, Sumi M, Hirata Y and Nagai R: Pioglitazone ameliorates endothelial dysfunction and restores ischemia-induced angiogenesis in diabetic mice. Biomed Pharmacother 62: 46-52, 2008

This work is licensed under a Creative Commons Attribution-NonCommercial-NoDerivatives 4.0 International (CC BY-NC-ND 4.0) License. 